\title{
ORIGINAL ARTICLE Codeine-induced hyperalgesia and allodynia: investigating the role of glial activation
}

\author{
JL Johnson ${ }^{1}$, PE Rolan ${ }^{1,2,3}$, ME Johnson ${ }^{4}$, L Bobrovskaya ${ }^{4}$, DB Williams ${ }^{4}$, K Johnson $^{5}$, J Tuke ${ }^{6}$ and MR Hutchinson ${ }^{7}$
}

\begin{abstract}
Chronic morphine therapy has been associated with paradoxically increased pain. Codeine is a widely used opioid, which is metabolized to morphine to elicit analgesia. Prolonged morphine exposure exacerbates pain by activating the innate immune tolllike receptor-4 (TLR4) in the central nervous system. In silico docking simulations indicate codeine also docks to MD2, an accessory protein for TLR4, suggesting potential to induce TLR4-dependent pain facilitation. We hypothesized codeine would cause TLR4dependent hyperalgesia/allodynia that is disparate from its opioid receptor-dependent analgesic rank potency. Hyperalgesia and allodynia were assessed using hotplate and von Frey tests at days 0, 3 and 5 in mice receiving intraperitoneal equimolar codeine $\left(21 \mathrm{mg} \mathrm{kg}^{-1}\right)$, morphine $\left(20 \mathrm{mg} \mathrm{kg}^{-1}\right)$ or saline, twice daily. This experiment was repeated in animals with prior partial nerve injury and in TLR4 null mutant mice. Interventions with interleukin-1 receptor antagonist (IL-1RA) and glial-attenuating drug ibudilast were assessed. Analyses of glial activation markers (glial fibrillary acid protein and CD11b) in neuronal tissue were conducted at the completion of behavioural testing. Despite providing less acute analgesia $(P=0.006)$, codeine induced similar hotplate hyperalgesia to equimolar morphine vs saline $(-9.5 \mathrm{~s}, P<0.01$ and $-7.3 \mathrm{~s}, P<0.01$, respectively), suggesting codeine does not rely upon conversion to morphine to increase pain sensitivity. This highlights the potential non-opioid receptor-dependent nature of codeineenhanced pain sensitivity-although the involvement of other codeine metabolites cannot be ruled out. IL-1RA reversed codeineinduced hyperalgesia $(P<0.001)$ and allodynia $(P<0.001)$, and TLR4 knock-out protected against codeine-induced changes in pain sensitivity. Glial attenuation with ibudilast reversed codeine-induced allodynia $(P<0.001)$, and thus could be investigated further as potential treatment for codeine-induced pain enhancement.
\end{abstract}

Translational Psychiatry (2014) 4, e482; doi:10.1038/tp.2014.121; published online 11 November 2014

\section{INTRODUCTION}

Opioid analgesics, used medicinally for millennia, remain vital in pain management. However, convincing preclinical and mounting clinical evidence suggests that long-term opioid use may paradoxically increase pain resulting in opioid-induced hyperalgesia. ${ }^{1}$ Opioid-induced hyperalgesia has been reported following the administration of a range of opioids, yet it has not been established if the 'weak' opioid codeine, can induce this phenomenon. Codeine-induced hyperalgesia is of particular interest, as in many regions codeine is available over-thecounter leading to widespread, unregulated consumption., ${ }^{2,3}$ Codeine is the most frequently used opioid in several European countries, ${ }^{4,5}$ and although most guidelines recommend only short-term codeine treatment, pharmacoepidemiological evidence indicates that $>10 \%$ of patients prescribed codeine consume more than the 120 defined daily doses per year, indicating chronic use. ${ }^{6}$ Codeine has low affinity for $\mu$-opioid receptors, compared with other opioid analgesics, ${ }^{7}$ and is considered a prodrug, dependent on transformation to morphine to relieve pain. ${ }^{8}$

Once absorbed, codeine undergoes partial O-demethylation to morphine via polymorphic cytochrome P450 isoenzyme 2D6 (CYP2D6). ${ }^{9,10}$ Most individuals convert $\sim 10 \%$ of an oral codeine dose to morphine. ${ }^{10}$ In mice, information regarding the metabolism of codeine is incomplete, yet O-demethylation of metoprolol, a typical human CYP2D6 substrate, is similar to that seen in human liver microsomes, ${ }^{11}$ providing evidence that BALB/ c mice are an acceptable model to assess clinically relevant codeine pharmacodynamics.

Numerous opioid-receptor-dependent neuronal mechanisms of opioid-induced hyperalgesia have been proposed (see Ossipov et $a .^{12}$ for review), however, substantial preclinical evidence establishes that morphine activates not only classical opioid receptors, but also toll-like receptor-4 (TLR4) on glia, triggering proinflammatory mediator release, initiating a cascade of events that enhance nociception. ${ }^{13}$ While neuronal morphine actions are analgesic, concurrent production of neuroexcitatory substances by glial cells (for example, astrocytes, microglia) counteracts this analgesia, to eventually increase pain. Thus, with increasing morphine dose and/or duration, TLR4-dependent glial reactivity increases reducing the analgesic efficacy and ultimately leading to allodynia and hyperalgesia. ${ }^{14}$

This TLR4-glial hypothesis has been established for morphine ${ }^{15}$ and oxycodone ${ }^{16}$ but remains to be tested for codeine. It is plausible that codeine may induce hyperalgesia indirectly by acting as a prodrug for morphine delivery. Insufficient evidence exists to allow the use of a codeine $\mathrm{O}$-demethylation inhibitor to test whether the conversion to morphine is solely responsible for

\footnotetext{
${ }^{1}$ Discipline of Pharmacology, School of Medical Sciences, University of Adelaide, Adelaide, SA, Australia; ${ }^{2}$ Pain and Anaesthesia Research Clinic, University of Adelaide, Adelaide, SA, Australia; ${ }^{3}$ Pain Management Unit, Royal Adelaide Hospital, Adelaide, SA, Australia; ${ }^{4}$ School of Pharmacy and Medical Sciences, University of South Australia, Adelaide, SA, Australia; ${ }^{5}$ Medicinova, San Diego, CA, USA; ${ }^{6}$ School of Mathematical Sciences, University of Adelaide, Adelaide, SA, Australia and ${ }^{7}$ Discipline of Physiology, School of Medical Sciences, University of Adelaide, Adelaide, SA, Australia. Correspondence: JL Johnson, Discipline of Pharmacology, School of Medical Sciences, Level 5, Medical School North, Frome Road, University of Adelaide, Adelaide, SA 5005, Australia. 
any hyperalgesia observed in mice. Instead, here we compared hyperalgesia precipitated by equimolar doses of codeine and morphine. Hypothesizing opioid-induced hyperalgesia to be dosedependent, ${ }^{17,18}$ if codeine were only able to facilitate pain once metabolized to morphine, then significantly less hyperalgesia would be expected in animals receiving codeine vs morphine, as only a minor proportion of the codeine dose is converted to morphine.

In silico docking simulations suggest that codeine docks to TLR4 accessory protein MD2, ${ }^{19}$ in a manner similar to morphine, ${ }^{15,20}$ indicating codeine has the potential to trigger TLR4-dependent pain enhancement. Owing to codeine's lower $\mu$-opioid receptor affinity, higher doses are required relative to morphine to produce equianalgesia. If codeine activates TLR4, greater glial activation could occur following equianalgesic codeine vs morphine, as a greater number of molecules must be administered to obtain the same therapeutic response. Thus, we hypothesize that the risk (hyperalgesia) to benefit (analgesia) ratio is greater for codeine compared with morphine.

\section{Objectives}

The objectives of the experiments presented in this manuscript were as follows: to determine whether chronic codeine administration induces hyperalgesia to the same degree as chronic morphine administration, to ascertain if partial nerve injury primes for codeine-induced hyperalgesia, to investigate the roles of proinflammatory cytokine interleukin-1 and TLR4 in the development of codeine-induced pain enhancement and finally to test the efficacy of a glial-attenuating agent in the reversal of codeineinduced hyperalgesia.

\section{MATERIALS AND METHODS}

Animals

Pathogen-free adult male wild-type BALB/c mice were obtained from the University of Adelaide Laboratory Animal Services (Adelaide, SA, Australia). Mice were housed in temperature $\left(18-21^{\circ} \mathrm{C}\right)$ and light-controlled $(12 \mathrm{~h}$ light/dark cycle; lights on at $0700 \mathrm{~h}$ ) rooms with standard rodent food and water available ad libitum. After arrival, the mice were allowed to acclimate to the facility for at least 5 days and were subsequently handled for a further 5 days before testing. All procedures were approved by the Animal Ethics Committee of the University of Adelaide and were conducted in accordance with the NHMRC Australian Code of Practice for the Care and Use of Animals for Scientific Purposes and the guidelines of the Committee for Research and Ethical Issues of International Association for the Study of Pain.

\section{Drugs}

All treatments were administered via intraperitoneal injection at a volume of $10 \mathrm{ml} \mathrm{kg}^{-1}$. Morphine hydrochloride (McFarlan Smith, Sydney, NSW, Australia) in $0.9 \%$ saline was administered at $20 \mathrm{mg} \mathrm{kg}^{-1}$ (base-corrected). Codeine phosphate (GlaxoSmithKline, Melbourne, VIC, Australia) in $0.9 \%$ saline, was administered at $21 \mathrm{mg} \mathrm{kg}^{-1}$ (base-corrected equimolar dose to morphine). Anakinra (Kineret, Amgen, Thousand Oaks, CA, USA), a recombinant, nonglycosylated form of the human interleukin-1 receptor antagonist (IL-1RA) in $0.9 \%$ saline was administered at $100 \mathrm{mg} \mathrm{kg}^{-1}$. Ibudilast (Medicinova, San Diego, CA, USA) in 35\% polyethelene glycol 400 (BDH Laboratory Supplies, Poole, England) in $0.9 \%$ saline was administered at $15 \mathrm{mg} \mathrm{kg}^{-1}$. As ibudilast was administered in a single intraperitoneal injection with concomitant codeine or morphine, the total dose volume for both drugs together was adjusted to $10 \mathrm{ml} \mathrm{kg}^{-1}$ for consistency between experiments. An equal volume of saline $0.9 \%$ was administered to control animals in Experiments 1, 2, 3 and 4, whereas control animals in Experiment 5 received $35 \%$ polyethylene glycol in $0.9 \%$ saline.

\section{Drug administration}

The morphine dose of $20 \mathrm{mg} \mathrm{kg}^{-1}$ used throughout the experiments outlined below was based upon that previously used in our laboratory to induce thermal hyperalgesia. The codeine dose was calculated to be equimolar to morphine, not equianalgesic. As briefly discussed in the introduction, only $\sim 10 \%$ of the codeine dose is thought to be metabolized to morphine in vivo, thus when codeine $21 \mathrm{mg} \mathrm{kg}^{-1}$ is administered, the animal will be exposed to substantially less morphine than when dosed with morphine $20 \mathrm{mg} \mathrm{kg}^{-1}$.

Experiments $1 a$ and $1 b$ : Assessment of codeine-induced hyperalgesia and allodynia and impact of prior partial nerve injury. In part 1a, wild-type mice were randomly allocated to receive codeine $(n=8)$, morphine $(n=8)$ or saline $(n=8)$ twice daily for 4 days. In part $1 \mathrm{~b}$, all mice underwent a modified version of the chronic constriction injury surgery to induce mild allodynia, as described below. Two weeks post surgery, wild-type mice were randomly allocated to receive codeine $(n=8)$, morphine $(n=8)$ or saline $(n=8)$ twice daily for 4 days. Behavioural assessments were conducted at baseline, before dosing, on day 3 and in the morning of day 5 .

Experiment 2: Comparison of acute analgesia between codeine and morphine. Wild-type mice $(n=8)$ were randomized to receive a single dose of codeine, morphine and saline on three respective testing days separated by 1-week washout periods. Hotplate testing was conducted three times and averaged to establish baseline sensitivity, and repeated at $15,30,40,50,60,75$ and $90 \mathrm{~min}$ post dose.

Experiment 3: Interleukin-1 receptor antagonist intervention. Wild-type mice were randomly allocated to a $2 \times 2$ design of 2 (codeine vs morphine; twice daily for 4 days) $\times 2$ (IL-1RA vs saline; morning of day 5, $30 \mathrm{~min}$ before behavioural testing) $n=8$ per group. Behavioural assessments were conducted at baseline, before dosing on day 3 and in the morning of day 5 .

Experiment 4: Assessment of codeine-induced hyperalgesia and allodynia in TLR4 null mutant (TLR4-/-) mice. TLR4 -/- mice were randomly allocated to receive codeine $(n=8)$, morphine $(n=8)$ or saline $(n=8)$ twice daily for 4 days. Behavioural assessments were conducted at baseline, before dosing on day 3 and in the morning of day 5 .

Experiment 5: Glial attenuating intervention. Wild-type mice were randomly allocated to a $2 \times 2$ design of 2 (codeine vs morphine; twice daily for 4 days) $\times 2$ (ibudilast vs PEG400; days 3 and 4 twice daily) $n=8$ per group. Behavioural assessments were conducted at baseline, before dosing, on day 3 and in the morning of day 5 .

\section{Partial nerve injury surgery}

The Grace model, ${ }^{21}$ a modified version of the chronic constriction injury model of sciatic nerve injury ${ }^{22}$ was performed at mid-thigh level of the left hind leg under isoflurane anaesthesia (3\% in oxygen). Briefly, the sciatic nerve was gently isolated with glass instruments and a single sterile chromic gut suture (cuticular 4-0 chromic gut, FS-2; Ethicon, Somerville, NJ, USA) was loosely tied around the sciatic nerve. The superficial muscle over the nerve was closed and three additional lengths of chromic gut were placed subcutaneously. The Grace model, in which a single chromic gut suture is placed around the sciatic nerve $(\mathrm{N})$ and three pieces of chromic gut are placed subcutaneously (S) is designated N1S3, and creates a mild pain state. $^{21}$ All the surgery was performed using aseptic surgical techniques with sterilized instruments. Animals were monitored postoperatively until ambulatory before being returned to their home cage and inspected daily for signs of infection. No such cases occurred in this study. Although rescue morphine analgesia was on hand to administer following the surgery if an adverse event occurred, no such additional analgesia was required.

\section{Behavioural testing}

All behavioural testing was conducted during the light phase of the light/ dark cycle and followed at least two habituations to the testing environment. The hotplate test and the von Frey test, two robust, wellestablished methods for assessing opioid analgesia and nociceptive sensitivity in rodents, were selected for use in this study as such tests are simple to conduct and repeated testing can be performed, allowing each animal to function as its own matched control. ${ }^{23}$

Allodynia assessments. The von Frey test was performed within the sciatic innervation region of both hind paws as previously described in detail. ${ }^{24}$ 
Briefly, mice were placed in individual cylindrical plastic cubicles $(10 \mathrm{~cm}$ $\mathrm{D} \times 15 \mathrm{~cm} \mathrm{H}$ ) with sufficient room to move freely on a wire mesh (6 $\mathrm{mm} \times 6 \mathrm{~mm}$ ) platform and allowed to acclimate for $\sim 20 \mathrm{~min}$. A logarithmic series of six calibrated von Frey monofilaments (Touch Test Sensory Evaluator Kit, St Louis, MO, USA), with bending forces that ranged from 0.02-0.4 g, were used to deliver the mechanical stimuli to the left and right hind paws in a random order. Each filament was applied to the left and right hind paw 10 times, and the number of paw-withdrawal responses elicited was recorded. Testing was conducted blind to treatment group allocation.

All animal assessments were conducted at baseline, before drug, dosing on day 3 of the experimental period and in the morning of day 5 of the experimental period. For animals in Experiment 2, additional assessments were conducted on days 3, 7, 11 and 14 post surgery, before randomization to treatment.

Hyperalgesia assessments. In all experiments, hotplate assessments were conducted at baseline, before morning drug dosing on day 3 and the morning of day 5 , after von Frey assessments. Mice were placed on a hotplate maintained at $50 \pm 0.2^{\circ} \mathrm{C}_{1}^{25}$ a clean glass cup was placed over the animal and the latency to paw withdrawal (seconds) was recorded. Mice had sufficient room to move freely while under the glass cup. Baseline withdrawal values were calculated from an average of three consecutive latencies, measured at $10 \mathrm{~min}$ intervals. A pre-determined cut-off time of $60 \mathrm{~s}$ was imposed to prevent tissue damage. ${ }^{25}$ Mice were immediately removed from the hotplate surface following the end of a trial due to pawwithdrawal response or elapsed cut-off time.

\section{Western blot analysis of GFAP and CD11b}

Four animals from each treatment group were anaesthetized with intraperitoneal sodium pentobarbital (Abbott Laboratories, Chicago, IL, USA) and transcardially perfused with phosphate-buffered saline to flush blood cells from the central nervous system tissue. The lumbar section ( $L 4$ to L6) of the spinal column and trigeminal ganglia were dissected out, immediately snap-frozen in liquid nitrogen and stored at $-70^{\circ} \mathrm{C}$ until processing. In preparation for the analysis, samples were immersed in cell lysis buffer containing $1 \%$ protease inhibitor cocktail (Sigma-Aldrich, Sydney, NSW, Australia, catalogue \# P8340), sonicated then centrifuged at 14000 r.p.m. for $5 \mathrm{~min}$. The supernatant was collected and the pellet discarded. Bicinchoninic acid (BCA) assays were performed to determine total protein concentration and subsequently the samples were diluted to allow loading of $30 \mu \mathrm{g}$ of protein per western blot well. Samples were run on an 8 or $10 \%$ sodium dodecyl sulphate-polyacrylamide gel and transferred to nitrocellulose using the wet transfer method. Membranes were immunoblotted with glial fibrillary acid protein (GFAP, 1:3000, Santa Cruz Biotechnology, Dallas, TX, USA, catalogue \#sc-6170) or cluster of differentiation molecule 11b (CD11b, 1:2000, Santa Cruz Biotechnology, catalogue \#sc-6614) antibodies overnight at $4{ }^{\circ} \mathrm{C}$. Blots were washed and left to incubate with appropriate secondary antibodies for $1 \mathrm{~h}$. Following a 1 min incubation with the enhanced chemiluminescence detection reagent, immunoblots were visualized using a LAS 4000 imaging system (GE Healthcare, Little Chalfont, UK) for the detection of chemiluminescent signals. The density of protein bands of interest were then quantified using
ImageQuant TL software (GE Healthcare). Subsequently membranes were washed and then immunoblotted with $\beta$-actin antibody (1:10 000, SigmaAldrich, catalogue \#A3854) as a marker of total protein loaded per each lane. GFAP and CD11b protein levels were normalized relative to $\beta$-actin levels.

\section{Statistical analysis}

All data are presented as mean \pm s.e.m. unless otherwise noted. Statistical analyses were performed using R via RStudio (Version 0.97.312, RStudio, Boston, MA, USA) ${ }^{26}$ and GraphPad Prism (Version 6, GraphPad Software, San Diego, CA, USA). Hotplate test data were analysed using linear mixed effects modelling with the R packages Ime 4,27 followed by simultaneous tests for general linear hypothesis and adjusted for multiple comparisons using Tukey contrasts, with adjusted $P$-values reported using the singlestep method. ${ }^{28}$ A one-way repeated measures analysis of variance (ANOVA), corrected with a post hoc Tukey's multiple comparisons test, ${ }^{26}$ was used to analyse differences in acute analgesia between the treatment groups in Experiment 2. For each von Frey test, von Frey filament number was plotted against percentage response (number of withdrawals per 10 filament applications $\times 10$ ), giving a slope and intercept for each animal at each test time point using the $\mathrm{R}$ package ggplot2. ${ }^{29}$ Slope represents percentage change in response as von Frey filament stiffness increases. A positive slope indicates a greater percentage response to high von Frey filament pressures vs low pressures, whereas a negative slope indicates a greater percentage response to low von Frey filament pressures vs high pressures, and as the slope approaches zero the percentage response to low and high von Frey filament pressures become similar. The intercept is an indicator of sensitivity to very low pressures; a greater intercept indicates greater allodynia elicited by low pressures. Slope and intercept were combined to form the allodynia outcome measure and analysed using multivariate ANOVA tests. ${ }^{26}$ For simplicity, only von Frey results for the left leg are presented as all the treatments and interventions were delivered systemically or performed on the left side. Western blot results were analysed using two-way ANOVA tests with Bonferroni post hoc tests to adjust for multiple comparisons. Correlations between western blot data and behavioural data were investigated using linear mixed effects modelling, ${ }^{27}$ followed by AIC stepwise model selection using the stepAIC function from the MASS library. ${ }^{30} P$-values $<0.5$ and $F$-values $>3$ were considered to indicate a significant difference. All R code employed during analysis is available upon request from the authors.

\section{RESULTS}

Experiments 1a and 1b: Assessment of codeine-induced hyperalgesia and allodynia and impact of prior partial nerve injury in wild-type mice.

Linear mixed effects modelling including data from both Experiments $1 \mathrm{a}$ and $1 \mathrm{~b}$ highlighted significant effects of drug $(F=12.6)$, day $(F=36.9)$ and surgery $(F=114.0)$ alone, as well as significant drug:day $(F=8.5)$ and day:surgery $(F=16.0)$ interaction effects on hyperalgesia in wild-type mice. Furthermore, as
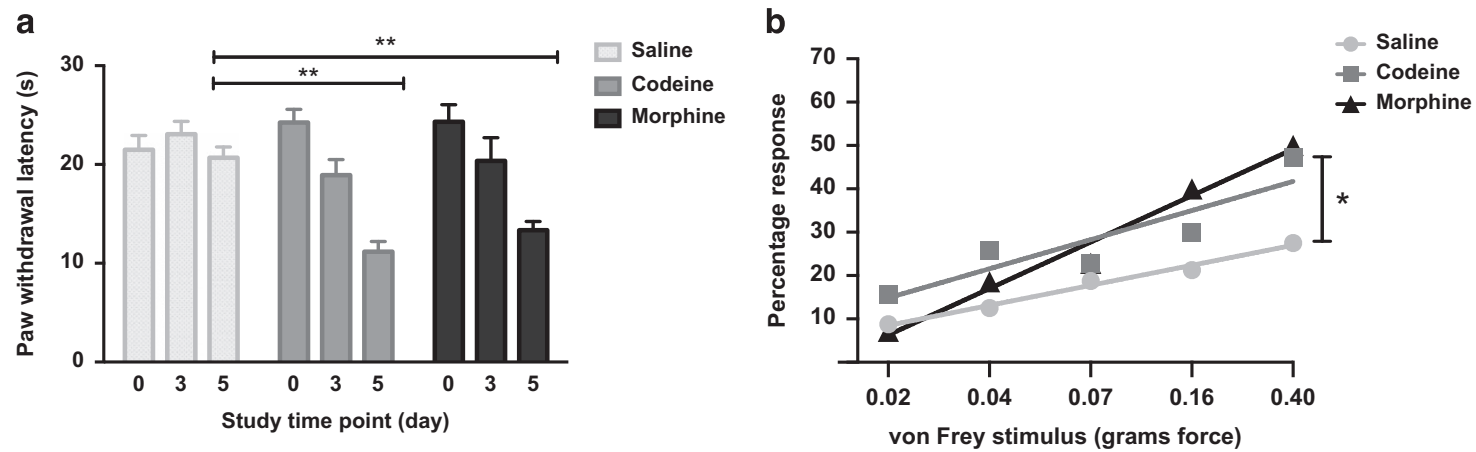

Figure 1. Experiment 1a: mice received intraperitoneal codeine $\left(21 \mathrm{mg} \mathrm{kg}^{-1}, n=8\right)$, morphine $\left(20 \mathrm{mg} \mathrm{kg}^{-1}, n=8\right)$ or saline $(n=8)$ twice daily for 4 days. Hyperalgesia (hotplate) and allodynia (von Frey) were measured on days 0, 3 and 5. (a) Codeine and morphine significantly reduced hotplate paw-withdrawal latency at day 5 vs saline. (b) There was a significant effect of drug on the development of allodynia in the codeine group (total allodynia was measured by calculating the slope and intercept for the plot of percentage response (number of paw withdrawals per 10 applications $x$ 10) vs von Frey stimulus for each group). ${ }^{*} P<0.05,{ }^{*} P<0.01$. 
illustrated in Figure 1a, Tukey post hoc analyses revealed that animals receiving codeine $21 \mathrm{mg} \mathrm{kg}^{-1}$ and morphine $20 \mathrm{mg} \mathrm{kg}^{-1}$ twice daily for 4 days displayed significantly reduced pawwithdrawal latency, indicative of hyperalgesia, on day 5 compared with saline-treated wild-type mice $(P<0.01$ and $P<0.01$, respectively). Paw-withdrawal latency was also reduced on day 5 vs baseline within the codeine $(P<0.001)$ and morphine $(P<0.001)$ groups. In Experiment $1 \mathrm{~b}$, partial nerve injury induced hyperalgesia at baseline in all groups, which was equivalent to that present in the codeine and morphine groups who did not undergo surgery on day 5 of the treatment. Basal hyperalgesia was present to such a degree that further decreases in latency in post-surgery animals receiving codeine $(P=0.5)$ or morphine $(P=0.9)$ on day 5 vs baseline, or between post-surgery mice receiving codeine $(P=0.4)$ and morphine $(P=0.2)$ vs saline on day 5 did not reach significance (data not shown). No differences were detected between codeine and morphine groups within Experiments 1a and $1 \mathrm{~b}$ on days 3 and 5 .

Multivariate ANOVA of all von Frey data from Experiments 1a and $1 \mathrm{~b}$ combined on day 5 highlighted overall significant effects of both drug $(P=0.007)$ and surgery $(P<0.001)$ on allodynia, indicating that both taking codeine or morphine, or undergoing surgery, increases sensitivity to non-noxious stimuli. In the codeine groups, drug $(P=0.03$, see Figure $1 \mathrm{~b})$ and surgery $(P<0.001)$ significantly influenced allodynia, yet there was no significant surgery:drug interaction $(P=0.91)$, demonstrating that codeineinduced allodynia did not differ between surgery and no-surgery animals. In the morphine groups, although surgery alone had a significant effect on allodynia $(P<0.001)$, drug $(P=0.08)$ did not contribute to allodynia until surgery was factored in, as indicated by a significant surgery:drug interaction $(P=0.04)$. This significant surgery:drug interaction demonstrates that the development of allodynia following morphine treatment differs in surgery animals compared with no-surgery animals. Animals who received morphine without surgery displayed lesser sensitivity to low von Frey filament pressures than morphine animals who underwent surgery; however, regardless of surgery status, both groups receiving morphine displayed a high response rate to high von Frey filament pressures.

Behavioural test results for codeine and morphine treatment groups were compared with saline controls on day 5, as well as their respective baseline findings, to control for learned or conditioned behaviour to the testing procedures. However, such factors are unlikely to influence results as no significant change in hotplate latency or response to von Frey filament stimulation was observed in the saline animals across the 5 days of testing.

Experiment 2: Comparison of acute analgesia between codeine $21 \mathrm{mg} \mathrm{kg}^{-1}$ and morphine $20 \mathrm{mg} \mathrm{kg}^{-1}$

Acute codeine administration at $21 \mathrm{mg} \mathrm{kg}^{-1}$ provided significantly less hotplate analgesia than morphine $20 \mathrm{mg} \mathrm{kg}^{-1}$ measured in terms of area under the time-effect (paw-withdrawal latency/ analgesia) curve from 0-90 min post dose, as shown in Figure 2.

Experiment 3: Interleukin-1 receptor antagonist intervention A significant overall effect of intervention (IL-1RA or saline) was detected in both hotplate $(P<0.001)$ and von Frey $(P<0.001)$ tests. As illustrated in Figure $3 a 1$, both codeine and morphine when given with saline only produced significant hyperalgesia $(P<0.001$ and $P<0.001$, respectively) at day 5 vs baseline. In codeine and morphine animals receiving IL-1RA before the final hyperalgesia assessment on day 5, paw-withdrawal latency began to return to baseline levels for both opioid groups, with morphine + IL-RA vs morphine+saline $(P<0.001)$ and codeine+IL-RA vs codeine+saline $(P<0.001)$ reaching significance. In the von Frey test, codeine and morphine, given with saline, established allodynia on day 5 vs baseline $(P<0.001$ and $P<0.001$,

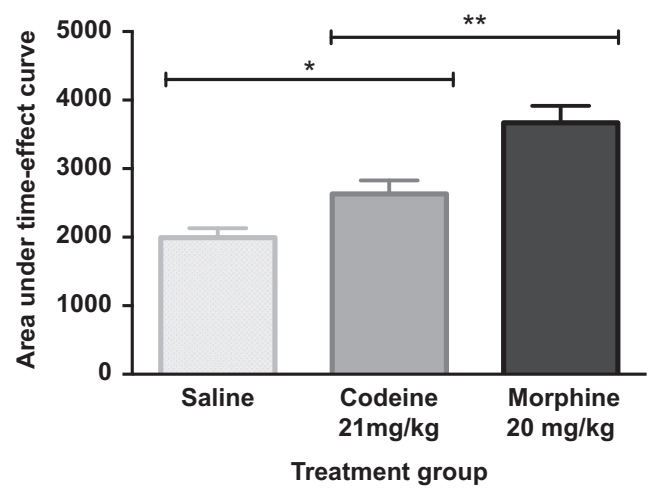

Figure 2. Experiment 2 (acute analgesia): mice $(n=8)$ were randomized to receive a single codeine $\left(21 \mathrm{mg} \mathrm{kg}^{-1}\right)$, morphine (20 mg kg $\left.{ }^{-1}\right)$ and saline dose on three separate occasions, separated by a washout period of 1 week. Hotplate testing was performed at baseline and 15,30,40,60, 60 and $75 \mathrm{~min}$ post dose and total analgesia produced over the 0-90 min testing period for each drug was calculated to give area under the time-effect (analgesia) curve (AUC). Codeine produced a greater AUC from 0-90 min post dose vs saline. Morphine produced a significantly greater AUC from 0 $90 \mathrm{~min}$ post dose vs an equimolar dose of codeine. ${ }^{*} P<0.05$, ${ }^{* *} P<0.01$

respectively). Partially established allodynia in codeine and morphine groups was abolished by IL-RA on day 5, as depicted in Figure $3 a 2$.

Experiment 4: Assessment of codeine-induced hyperalgesia and allodynia in TLR4 - / - mice

Incorporating data from the no-surgery Experiment 1a mice, linear mixed effects modelling found significant effects of genotype (TLR4 - / - or wild type) alone ( $F=7.6)$, as well as significant drug: genotype $(F=3.002)$ and genotype:day $(F=21.2)$ interactions effects on hyperalgesia. Tukey post hoc analyses confirmed no significant differences in paw-withdrawal latency in the hotplate test between treatment groups on day 5 in TLR4 - / - animals (see Figure 3b1). Similarly, multivariate ANOVA revealed a significant effect of genotype $(P<0.0001)$ on allodynia at day 5 and demonstrated that TLR4-/- mice were protected against changes in pain sensitivity in all treatment groups as shown in Figures $3 b 2$, b3 and b4.

Experiment 5: Glial attenuating intervention

A significant overall effect of intervention (ibudilast or vehicle) was detected in both hotplate $(P=0.002)$ and von Frey $(P=0.003)$ tests. As shown in Figure $3 \mathrm{c} 1$, ibudilast significantly increased pawwithdrawal latency vs vehicle in the morphine group $(P=0.035)$. The trend of hyperalgesia reversal following ibudilast in the codeine group did not reach significance vs vehicle $(P=0.246)$. Codeine and morphine groups receiving vehicle on days 3 and 4, in addition to their respective opioids, displayed allodynia $(P<0.001$ and $P=0.009$, respectively) at day 5 vs baseline. Established allodynia in codeine and morphine groups was abolished by ibudilast on day 5, as illustrated in Figure $3 c 2$.

\section{6 western blot analysis of GFAP and CD11b}

Following final behavioural testing bilateral trigeminal ganglion tissue and the lumbar section of the spinal cord were dissected and prepared for western blot analysis to investigate levels of markers associated with reactivity of glial cells. Levels of CD11b, an adhesion molecule marker for active macrophages and microglia $^{31,32}$ and GFAP, an increase in which accompanies the 
a1

$\square$ Codeine + saline $\square$ Morphine + saline 드 Codeine + IL-1RA Morphine + IL-1RA

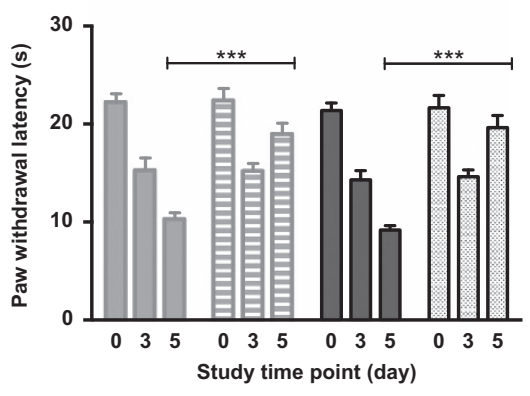

b1

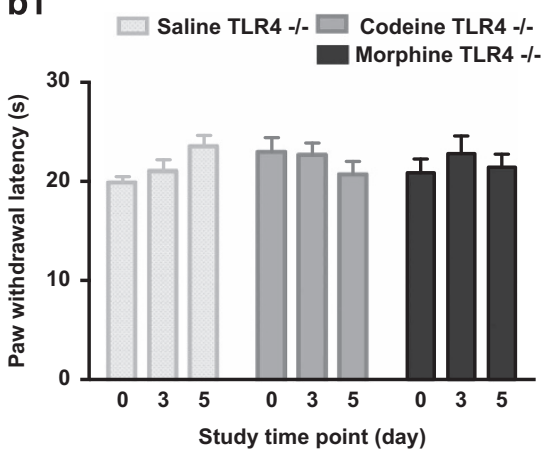

b3

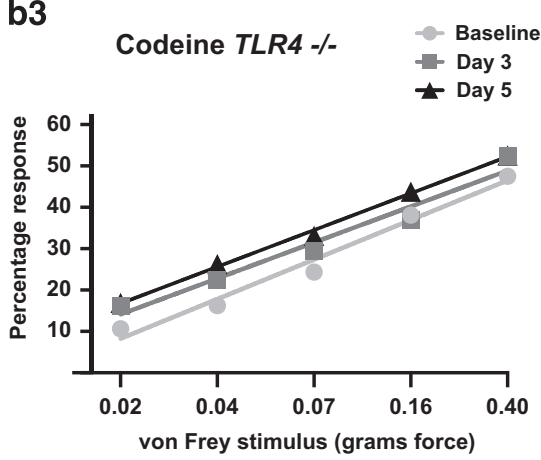

c1

$\square$ Codeine + ibudilast $\square$ Morphine + ibudilas 트 Codeine + vehicle Morphine + vehicle

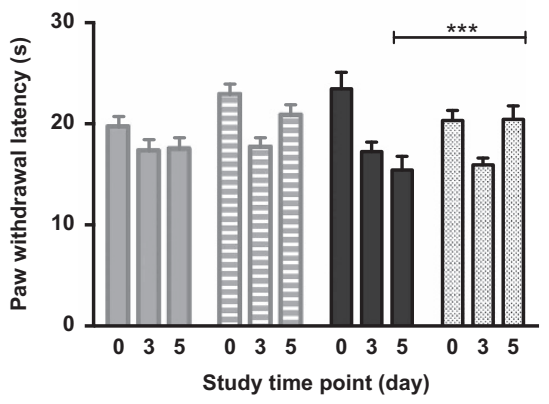

reactive response of astrocytes and satellite glial cells after exposure to an insult, ${ }^{33-35}$ were quantified.

When all drug treatment groups were taken together, partial nerve injury surgery before opioid administration significantly increased GFAP expression in the trigeminal ganglion $(P<0.01)$ and spinal cord $(P=0.02)$. However, no overall effect of drug was a2

- Codeine + saline - Morphine + saline

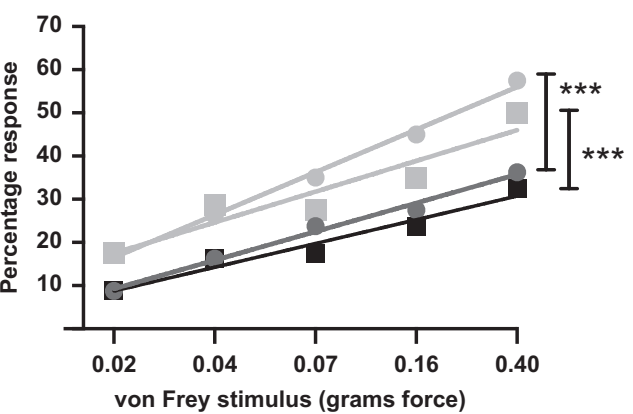

b2

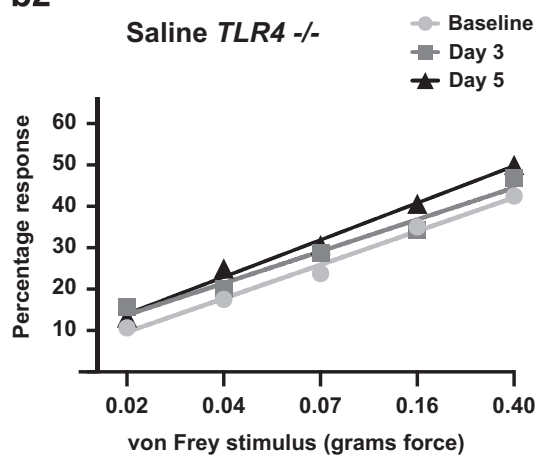

b4

Morphine TLR4 -/ $\quad$ Baseline

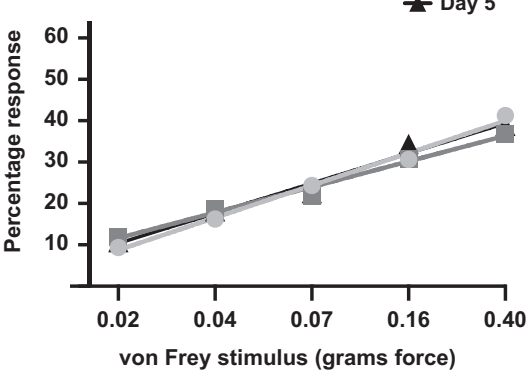

c2

- Codeine + vehicle Morphine + vehicle

- Codeine + ibudilast + Morphine + ibudilast

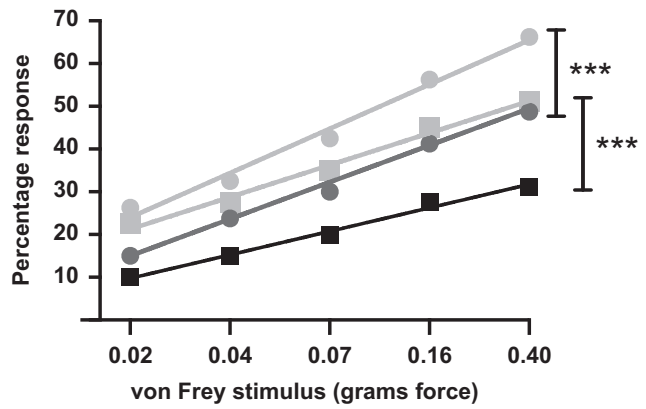

detected $(P=0.3)$. Significant effects of both surgery $(P=0.02)$ and drug $(P<0.01)$, and a surgery:drug $(P<0.01)$ interaction were found on CD11b levels in the spinal cord, although these appear to be driven largely by a difference within the codeine group. Drug also had a significant overall effect on CD11b in the trigeminal ganglion $(P<0.01)$. In Experiments $1 \mathrm{a}$ and $1 \mathrm{~b}$, linear 
Figure 3. (a) Experiment 3: mice received intraperitoneal (i.p.) codeine $\left(n=16,21 \mathrm{mg} \mathrm{kg}^{-1}\right)$ or morphine $\left(n=16,20 \mathrm{mg} \mathrm{kg}{ }^{-1}\right)$ twice daily for 4 days. Hyperalgesia (hotplate) and allodynia (von Frey) were measured on days 0,3 and 5 . Thirty minutes before assessments on day 5 , half of the mice in each drug group received i.p. interleukin-1 receptor antagonist (IL-1RA, $100 \mathrm{mg} \mathrm{kg}^{-1}$ ) and the remaining half received saline. (a1) IL-1RA abolished decreases in paw-withdrawal latency in both the codeine and morphine groups at day 5 vs groups receiving saline. (a2) IL-1RA significantly attenuated allodynia induced by codeine and morphine on day 5 vs saline in the left hind paw. (b) Experiment 4: toll-like receptor-4 null mutant mice received i.p. codeine $\left(n=8,21 \mathrm{mg} \mathrm{kg}^{-1}\right)$, morphine $\left(n=8,20 \mathrm{mg} \mathrm{kg}^{-1}\right)$ or saline $(n=8)$ twice daily for 4 days. Hyperalgesia (hotplate) and allodynia (von Frey) were measured on days 0,3 and 5. (b1) Codeine $(P=0.996)$ and morphine $(P>0.99)$ did not alter hotplate paw-withdrawal latency at day 5 vs baseline. Allodynia did not change significantly over time in (b2) saline $(P=0.09)$, (b3) codeine $(P=0.051)$ or (b4) morphine $(P=0.21)$ groups. (c) Experiment 5: mice received i.p. codeine $\left(n=16,21 \mathrm{mg} \mathrm{kg}{ }^{-1}\right)$ or morphine $(n=16$, $20 \mathrm{mg} \mathrm{kg}^{-1}$ ) twice daily for 4 days. On days 3 and 4 , half of the mice in each group received i.p. ibudilast $\left(15 \mathrm{mg} \mathrm{kg}{ }^{-1}\right)$ and the remaining half received vehicle twice daily. Hyperalgesia (hotplate) and allodynia (von Frey) were measured on days 0,3 and 5 . (c1) Ibudilast reversed decreases in hotplate paw-withdrawal latency in morphine but not codeine $(P=0.2)$ animals at day 5 vs groups receiving saline in addition to their respective opioid. (c2) Ibudilast significantly attenuated allodynia induced by codeine and morphine in the von Frey test on day 5 vs saline in the left hind paw. Total allodynia was measured by calculating the slope and intercept for the plot of number of paw withdrawals per 10 applications vs von Frey stimulus for each group. ${ }^{* * * P}<0.001$.

4

Table 1. Fold change between treatment groups and comparator controls in glial fibrillary acidic protein (GFAP) and cluster of differentiation molecule $11 \mathrm{~b}(\mathrm{CD} 11 \mathrm{~b})$ levels relative to $\beta$-actin, in the spinal cord and trigeminal ganglion.

\begin{tabular}{llcccc}
\hline \multirow{2}{*}{ Treatment group } & Comparator group & \multicolumn{3}{c}{ Fold change (P-value) } \\
\cline { 3 - 6 } & & Spinal cord GFAP & Spinal cord CD11b & Trigeminal ganglion GFAP & Trigeminal ganglion CD11b \\
\hline Codeine only & Saline only & $0.93(>0.99)$ & $1.59(<0.01)^{* *}$ & $0.60(0.14)$ & $0.25(<0.01)^{* *}$ \\
Morphine only & Saline only & $0.82(>0.99)$ & $0.73(0.23)$ & $0.58(>0.12)$ & $0.67(0.35)$ \\
Saline TLR4 - /- & Saline only & $1.47(0.32)$ & $0.46(<0.01)^{* *}$ & $6.33(0.02)^{*}$ & Unavailable \\
Codeine TLR4 -/- & Saline TLR4 -/- & $0.60(0.15)$ & $0.97(>0.99)$ & $0.83(>0.91)$ & $0.61(0.03)^{*}$ \\
Morphine TLR4 -/- & Saline TLR4 -/- & $0.43(0.03)^{*}$ & $0.74(0.56)$ & $0.25(>0.07)$ & $1.03(0.99)$ \\
Codeine+ibudilast & Codeine+vehicle & $1.32(0.29)$ & $1.66(0.14)$ & $0.06(0.01)^{*}$ & $0.18(<0.01)^{* *}$ \\
Morphine+ibudilast & Morphine+vehicle & $1.34(0.48)$ & $1.17(0.75)$ & $1.78(>0.99)$ & $1.78(0.44)$ \\
\hline
\end{tabular}

Abbreviation: TLR4, Toll-like receptor-4. Codeine only, codeine TLR4-/-, codeine+ibudilast and codeine+vehicle animals received codeine intraperitoneal (i.p.) $21 \mathrm{mg} \mathrm{kg}^{-1}$ twice daily for 4 days. Morphine only, morphine TLR4-/-, morphine+ibudilast and morphine+vehicle animals received morphine i.p. $20 \mathrm{mg} \mathrm{kg}^{-1}$ twice daily for 4 days. Saline only and saline TLR4 - / - animals received i.p. saline (equal volume to opioids) twice daily for 4 days. Codeine +ibudilast and morphine+ibudilast received i.p. ibudilast $15 \mathrm{mg} \mathrm{kg}^{-1}$ (in $35 \%$ polyethelene glycol) twice daily on days 3 and 4 , in addition to their respective opioids. Codeine+vehicle and morphine+vehicle animals received i.p. 35\% polyethelene glycol twice daily on days 3 and 4 , in addition to their respective opioids. All tissues samples were obtained on day 5 of the experimental protocols. Values $<1$ represent a reduction in marker level vs comparator whereas values $>1$ represent an increase relative to comparator. ${ }^{a}$ Unable to quantitate due to poor blot quality.

modelling with stepwise model selection by AIC demonstrates that spinal cord GFAP, CD11b and the interaction between spinal cord GFAP and CD11b are able to predict $82 \%$ of the variation in hotplate test scores in the morphine groups, and $35 \%$ of hotplate test score variability in the codeine groups, whereas no model based upon the spinal cord markers was able to account for hotplate test variability in the saline-treated mice.

Within TLR4-/- animals, codeine and morphine did not increase GFAP or CD11b at either site assessed. The TLR4-/mice displayed reduced $C D 11 \mathrm{~b}$ levels in the spinal cord, yet compared with wild-type animals, spinal GFAP was not altered $(P=0.5)$, and, in the trigeminal ganglion, CD11b $(P<0.01)$ and GFAP $(P<0.01)$ appeared elevated. Intervention with ibudilast did not bring about any differences in GFAP or CD11b in the spinal cord; yet in the trigeminal ganglion, codeine produced significant increases in GFAP $(P<0.01)$ and CD11b $(P<0.01)$ vs morphine, which were abolished following ibudilast administration. A summary of the relevant pair-wise comparisons in glial activation markers throughout all experiments is presented in Table 1 (Specific comparisons between partial nerve injury+drug and drug-only (no surgery) animals are not presented because no differences in hyperalgesia or allodynia were observed after drug treatment). Representative western blot images are presented in Figure 4.

\section{DISCUSSION}

In this study, we have demonstrated that codeine is able to induce hyperalgesia and allodynia to the same degree as equimolar morphine. This suggests that codeine may not rely solely upon metabolism to morphine to produce hyperalgesia, as if it was only the morphine metabolite of codeine causing pain sensitivity changes; significantly less hyperalgesia and allodynia would be expected in the codeine group, given that only a small proportion of the codeine dose is converted to morphine. The possibility that codeine is largely being converted to morphine in this mouse strain is unlikely as the hotplate test confirmed that codeine provides significantly less acute analgesia over $90 \mathrm{~min}$ post dose than morphine at an equimolar dose in male BALB/c mice, however, we cannot rule out the involvement of other codeine metabolites in the development of increased pain sensitivity. TLR4 and IL-1 $\beta$ appear to have important roles in the development of hyperalgesia as genetic lack of TLR4 and administration of an IL-1RA both abolished codeine-induced increases in pain sensitivity. Furthermore, attenuating glial activation with ibudilast reversed partially established codeine-induced allodynia.

To model the clinical situation in which opioids are administered to patients with an increased basal pain state, we first performed partial nerve injury surgery to prime animals before codeine administration. Relative to the hypothesized involvement of glial cells in morphine-induced hyperalgesia, we expected surgery to first prime the glial cells, triggering them to respond faster with greater magnitude, when subsequently activated following opioid administration. ${ }^{36}$ We found that surgery produced hyperalgesia and allodynia at baseline, and although codeine and morphine animals displayed a trend towards a further increase in pain sensitivity, this did not reach significance, likely owing to a floor effect of the behavioural testing employed. 
a

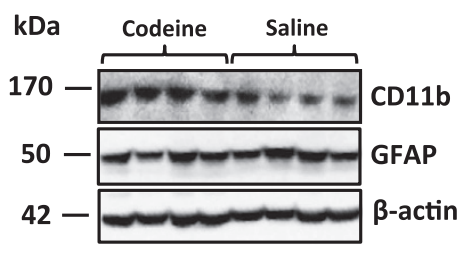

b

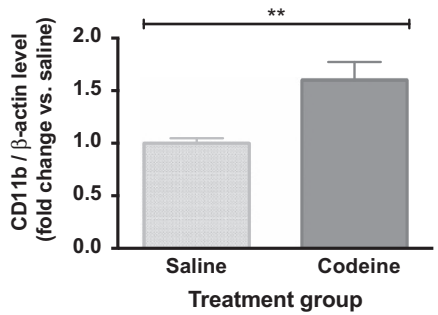

C

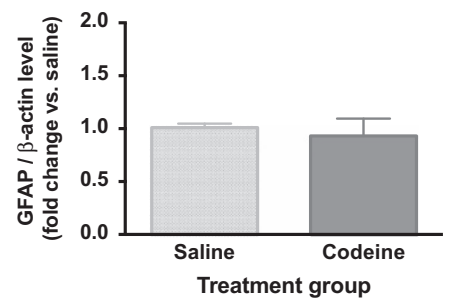

Figure 4. (a) Representative western blot images for cluster of differentiation molecule $11 \mathrm{~b}$ (CD11b), glial fibrillary acid protein (GFAP) and $\beta$ actin. Shows samples of the lumbar spinal cord of animals receiving intraperitoneal codeine ( $\left.21 \mathrm{mg} \mathrm{kg}^{-1}\right)$ or saline, twice daily for 4 days. Tissue samples collected on day 5 of the experimental protocol. (b) Fold change in lumbar spinal cord CD11b, normalized for $\beta$-actin, in animals receiving codeine $\left(21 \mathrm{mg} \mathrm{kg}^{-1}\right)$ compared with saline controls; corresponds with band at $170 \mathrm{kDa}$ in a. (c) Fold change in lumbar spinal cord GFAP, normalized for $\beta$-actin, in animals receiving codeine $\left(21 \mathrm{mg} \mathrm{kg}^{-1}\right)$ compared with saline controls; corresponds with band at $50 \mathrm{kDa}$ in a. ${ }^{* *} P<0.01$.

As in prior experiments, ${ }^{21,37}$ partial nerve injury surgery increased the overall levels of a marker of astrocyte activation, $\mathrm{GFAP}^{38}$ in the lumbar spinal cord. Administering morphine post surgery further elevated lumbar astrocyte activation, concurring with the results reported by Raghavendra et al., ${ }^{37}$ who found a greater magnitude of astrocyte activation following chronic morphine administration in nerve-injured compared with shamoperated rats, yet the same increase was not observed when codeine was given post surgery. Interestingly, unlike previous studies of morphine, ${ }^{37,39,40}$ we did not observe an increase in markers of glial activation in no-surgery animals receiving morphine, nor did we find increased glial activation markers in animals receiving chronic codeine, as compared with control animals. The significant hyperalgesia and allodynia present could, however, result from glial activation that was not captured by our tissue collection time point or proinflammation without phenotypic glial cell surface expression changes. While pain behaviour in codeine and morphine animals was comparable, and both groups were sensitive to the same pharmacological interventions (indicating similar mediators are at play), linear modelling suggests that molecular changes, inferred from the phenotypic cellular expression markers, are not identical. Although cellular surface markers represent only surrogates of activity, it is clear the processes underlying opioid-induced changes in pain sensitivity, resulting from the summation of $\mu$-opioid receptor and TLR4 signalling cascades, are indeed complex, particularly following codeine administration. Further studies with greater group numbers for protein analysis and multiple tissue collection time points may assist in clarifying proteomic results.

Toll-like receptor-4, which detects 'danger signals' and microbial associated molecular patterns-such as lipopolysaccharide found in the cell wall of gram-negative bacteria-is a key modulator in innate immune system activation. ${ }^{41}$ In our study, mice lacking TLR4 were protected against codeine-induced hyperalgesia and allodynia. Protection against glial activation in TLR4 $-/-$ mice could not be confirmed as western blot results were inconclusive, however, behavioural findings in TLR4 - / - mice were robust and agree with previous evidence that pharmacological blockade of TLR4 using (+)-naloxone is able to significantly attenuate paradoxical morphine-induced increases in pain sensitivity. ${ }^{20}$

Once activated, glial cells release a range of proinflammatory mediators such as the inflammatory cytokine IL-1 $\beta$. Again aligning with a glial activation hypothesis, we have demonstrated that pharmacological antagonism of the IL-1 receptor using anakinra is sufficient to reverse codeine-induced hyperalgesia and allodynia. These results also agree with literature indicating systemically delivered IL-1RA enters the central nervous system ${ }^{42,43}$ and is able to reinstate morphine analgesia in animals that display opioid tolerance, a phenomenon which has previously been described as a behavioural manifestation of opioid-induced hyperalgesia. ${ }^{44}$
Treatment with the glial-attenuating agent ibudilast ${ }^{45}$ reversed codeine-induced allodynia. Ibudilast's pharmacological actions include inhibition of macrophage migration inhibitory factor ${ }^{46}$ and phosphodiesterase 4 and 10 (and to a less extent 3 and 11). ${ }^{47}$ Both these inhibitory actions are thought to contribute to its glialattenuating ability. ${ }^{48}$ Previously ibudilast has been shown to potentiate the analgesic potency of morphine in tolerant rodents. $^{39}$ Given that blocking IL-1 reversed changes in pain sensitivity, and ibudilast has been shown to prevent the release of IL-1 from microglial cells, ${ }^{49}$ it is plausible that the reversal of opioid-induced allodynia by ibudilast is brought about either directly or indirectly through glial modulation. As a phosphodiesterase inhibitor vascular reactivity, and therefore drug distribution, could be altered following ibudilast administration. However, it is unlikely that altered distribution accounts for the reduction in morphine-induced hyperalgesia observed in this study as previous experiments have demonstrated that ibudilast co-administration does not alter morphine pharmacokinetics. ${ }^{39}$ Furthermore, multiple studies have established that ibudilast co-administration potentiates morphine analgesia, the opposite of what would be expected if ibudilast resulted in reduced distribution of morphine to the central nervous system. ${ }^{39,50}$

Although ibudilast has previously been reported to produce slight sedation, reduced locomotor activity ${ }^{50}$ and reduced sensitivity to touch, ${ }^{51}$ it is unlikely that these adverse effectswhich occur early (within 30 min of dosing) and are transient ${ }^{51}$ have influenced results, as all testing in our study was performed $16 \mathrm{~h}$ post ibudilast dose. While we could not confirm that ibudilast reduced the activation of lumbar spinal glial cells (which would mediate hind paw allodynia), a reduction in glial-mediated inflammation, independent of the cell surface markers assessed, could account for our results. In the trigeminal ganglion, ibudilast significantly reversed substantial codeine-induced satellite glial cell activation, indicating that the trigeminal ganglion glial cells may be particularly sensitive to the glial-activating effects of codeine, and ibudilast may be useful in attenuating activation at this site.

We are confident that our behavioural results are reproducible in male BALB/c mice, yet additional studies are required to determine generalizability to females and other strains of mice, as large variability in opioid response has been reported between female and male rodents ${ }^{52}$ and among different mice strains. ${ }^{53}$ Regardless, results from previous preclinical studies investigating opioid-induced hyperalgesia following morphine, methadone and buprenorphine dosing have been replicated in human trials, indicating that studies such as this do have positive predictive value in the clinic. In these experiments, pain sensitivity was only assessed the morning after opioid administration ( 16h postdose), thus the hyperalgesia and allodynia displayed may be related to processes involved in opioid withdrawal. In future 
studies, additional testing shortly after opioid administration would be of interest to determine if opioid tolerance was also present.

The observation of equal hyperalgesia following equimolar codeine and morphine administration has important clinical implications, as although both drugs appear to increase pain to the same degree, codeine provides only around one-tenth of the analgesia that morphine provides when equal doses are given, thus the risks:benefit ratio may be higher for codeine than for morphine. One example of a clinical pain state that is frequently worsened following codeine use is medication overuse headache, in which patients with an underlying primary headache disorder, progress to experience chronic daily or near-daily headache. ${ }^{54} \mathrm{We}$ have previously hypothesized that glial priming due to recurrent headaches may be responsible for the specific susceptibility to opioid-induced chronic headache observed in patients with preexisting headache conditions. ${ }^{19}$ Given that the trigeminal ganglion is of particular importance in the pathophysiology of migraine, the exaggerated satellite glial activation observed in the ibudilast intervention experiment suggests that codeine may be particularly detrimental in the management of this condition, and additional studies that include assessment of facial allodynia could provide a useful behavioural correlate to aid in interpreting protein analysis results.

\section{CONCLUSIONS}

This study provides the first preclinical evidence that repeated doses of codeine, similar to morphine, are able to induce both hyperalgesia and allodynia. Mechanistically, increased sensitivity to pain following codeine administration may occur as a result of TLR4 activation-dependent proinflammatory cytokine release by glial cells. Glial attenuation with agents such as ibudilast, for which the clinical safety is already established, may prove to be of use in the clinical management of opioid-induced hyperalgesia.

\section{CONFLICT OF INTEREST}

KJ was an employee at Medicinova, a biopharmaceutical company investigating ibudilast, while this study was conducted but no longer has material conflicts of interest to declare. The remaining authors declare no conflict of interest.

\section{ACKNOWLEDGMENTS}

We thank Lauren Nicotra for training the study staff to perform the required experimental procedures, Natalie Morrall and Dhanushika Ratnayake for their assistance with animal handling and tissue collection and Dr Daniel Barrett for his advice regarding statistical analysis. JLJ and MEJ are recipients of the Australian Postgraduate Award. MRH is the recipient of an Australian Research Council Research Fellow (DP110100297).

\section{REFERENCES}

1 Fishbain DA, Cole B, Lewis JE, Gao J, Rosomoff RS. Do opioids induce hyperalgesia in humans? An evidence-based structured review. Pain Med 2009; 10: 829-839.

2 MacFadyen L, Eadie D, McGowan T. Community pharmacists' experience of overthe-counter medicine misuse in Scotland. J R Soc Promot Health 2001; 121: 185. 3 Murnion BP. Combination analgesics in adults. Aust Prescr 2010; 33: 113-115.

4 Hamunen K, Laitinen-Parkkonen P, Paakkari P, Breivik H, Gordh T, Jensen NH et al. What do different databases tell about the use of opioids in seven European countries in 2002? Eur J Pain 2012; 12: 705-715.

5 Hamunen K, Paakkari P, Kalso E. Trends in opioid consumption in the Nordic countries 2002-2006. Eur J Pain 2009; 13: 954-962.

6 Fredheim OMS, Skurtveit S, Moroz A, Breivik H, Borchgrevink PC. Prescription pattern of codeine for non-malignant pain: a pharmacoepidemiological study from the Norwegian Prescription Database. Acta Anaesthesiol Scand 2009; 53: 627-633.

7 Coller J, Christrup L, Somogyi A. Role of active metabolites in the use of opioids. Eur J Clin Pharmacol 2009; 65: 121-139.
8 Eckhardt K, Li S, Ammon S, Schänzle G, Mikus G, Eichelbaum M et al. Same incidence of adverse drug events after codeine administration irrespective of the genetically determined differences in morphine formation. Pain 1998; 76: 27-33.

9 Holmquist G. Opioid metabolism and effects of cytochrome P450. Pain Med 2009; 10: S20-S29.

10 Vree T, Verwey-Van Wissen C. Pharmacokinetics and metabolism of codeine in humans. Biopharm Drug Dispos 1992; 13: 445-460.

11 Adams M, Lennard MS, Otton SV, Tucker GT, Woods HF. Assessment of the mouse as an experimental model for studying polymorphic oxidation of the sparteine/ debrisoquine type. Biochem Pharmacol 1991; 42: 947-949.

12 Ossipov MH, Lai J, King T, Vanderah TW, Porreca F. Underlying mechanisms of pronociceptive consequences of prolonged morphine exposure. Biopolymers 2005; 80: 319-324.

13 Hutchinson MR, Bland ST, Johnson KW, Rice KC, Maier SF, Watkins LR et al. Opioid-induced glial activation: mechanisms of activation and implications for opioid analgesia, dependence, and reward. ScientificWorldJournal 2007; 7: 98-111.

14 Hutchinson MR, Coats BD, Lewis SS, Zhang Y, Sprunger DB, Rezvani N et al. Proinflammatory cytokines oppose opioid-induced acute and chronic analgesia. Brain Behav Immun 2008; 22: 1178-1189.

15 Hutchinson MR, Lewis SS, Coats BD, Rezvani N, Zhang Y, Wieseler JL et al. Possible involvement of toll-like receptor 4 /myeloid differentiation factor- 2 activity of opioid inactive isomers causes spinal proinflammation and related behavioral consequences. Neuroscience 2010; 167: 880-893.

16 Hutchinson MR, Northcutt AL, Hiranita T, Wang X, Lewis SS, Thomas J et al. Opioid activation of toll-like receptor 4 contributes to drug reinforcement. $J$ Neurosci 2012; 32: 11187-11200.

17 Célèrier $E$, Rivat $C$, Jun $Y$, Laulin JP, Larcher A, Reynier $P$ et al. Long-lasting hyperalgesia induced by fentanyl in rats: preventive effect of ketamine. Anesthesiology 2000; 92: 465-472.

18 Jacquet $Y F$, Lajtha $A$. Morphine action at central nervous system sites in rat: analgesia or hyperalgesia depending on site and dose. Science 1973; 182: 490-492.

19 Johnson JL, Hutchinson MR, Williams DB, Rolan P. Medication-overuse headache and opioid-induced hyperalgesia: a review of mechanisms, a neuroimmune hypothesis and a novel approach to treatment. Cephalalgia 2013; 33: 52-64.

20 Hutchinson MR, Zhang Y, Shridhar M, Evans JH, Buchanan MM, Zhao TX et al. Evidence that opioids may have toll-like receptor 4 and MD-2 effects. Brain Behav Immun 2010; 24: 83-95.

21 Grace PM, Hutchinson MR, Manavis J, Somogyi AA, Rolan PE. A novel animal model of graded neuropathic pain: utility to investigate mechanisms of population heterogeneity. J Neurosci Methods 2010; 193: 47-53.

22 Bennett GJ, Xie YK. A peripheral mononeuropathy in rat that produces disorders of pain sensation like those seen in man. Pain 1988; 33: 87-107.

23 Barrot M. Tests and models of nociception and pain in rodents. Neuroscience 2012 211: 39-50.

24 Nicotra L, Tuke J, Grace P, Rolan PE, Hutchinson MR. Sex differences in mechanical allodynia: how can it be preclinically quantified and analysed? Front Behav Neurosci 2014; 8: 40.

25 Ankier SI. New hot plate tests to quantify antinociceptive and narcotic antagonist activities. Eur J Pharmacol 1974; 27: 1-4.

26 R Core Team. R: A Language and Environment for Statistical Computing. R Foundation for Statistical Computing: Vienna, Austria, 2013; http://www.R-project.org/.

27 Bates D, Maechler M, Bolker B, Walker S Ime4: linear mixed-effects models using Eigen and S4. R package version 1.0-5 2013; http://CRAN.R-project.org/package = Ime4.

28 Hothorn T, Bretz F, Westfall P. Simultaneous inference in general parametric models. Biom J. 2008; 50: 346-363.

29 Wickham H. The split-apply-combine strategy for data analysis. J Stat Softw 2011; 40: 1-29.

30 Venables WN, Ripley BD. Modern Applied Statistics with S, 4th edn. Springer: New York, NY, USA, 2002.

31 Glenn JA, Sonceau JB, Wynder HJ, Thomas WE. Histochemical evidence for microglia-like macrophages in the rat trigeminal ganglion. J Anat 1993; 183: 475-481.

32 Aita M, Byers MR, Chavkin C, Xu M. Trigeminal injury causes kappa opioiddependent allodynic, glial and immune cell responses in mice. Mol Pain 2010; 6: 8 .

33 Erickson PA, Feinstein SC, Lewis GP, Fisher SK. Glial fibrillary acidic protein and its mRNA: ultrastructural detection and determination of changes after CNS injury. J Struct Biol 1992; 108: 148-161.

34 Middeldorp J, Hol EM. GFAP in health and disease. Prog Neurobiol 2011; 93: 421-443.

35 Hanani M. Satellite glial cells in sensory ganglia: from form to function. Brain Res Brain Res Rev 2005; 48: 457-476. 
36 Perry VH, Hume DA, Gordon S. Immunohistochemical localization of macrophages and microglia in the adult and developing mouse brain. Neuroscience 1985; 15 : 313-326.

37 Raghavendra V, Rutkowski MD, DeLeo JA. The role of spinal neuroimmune activation in morphine tolerance/hyperalgesia in neuropathic and shamoperated rats. J Neurosci 2002; 22: 9980-9989.

38 Torre ER, Lothman E, Steward O. Glial response to neuronal activity: GFAP-mRNA and protein levels are transiently increased in the hippocampus after seizures. Brain Res 1993; 631: 256-264.

39 Hutchinson M, Lewis SS, Coats B, Skyba DA, Crysdale N, Berkelhammer DL et al. Reduction of opioid withdrawal and potentiation of acute opioid analgesia by systemic AV411 (ibudilast). Brain Behav Immun 2009; 23: 240-250.

40 Song P, Zhao ZQ. The involvement of glial cells in the development of morphine tolerance. Neurosci Res 2001; 39: 281-286.

$41 \mathrm{Li} \mathrm{Q}$. Antagonists of toll like receptor 4 maybe a new strategy to counteract opioid-induced hyperalgesia and opioid tolerance. Med Hypotheses 2012; 79: 754-756.

42 Greenhalgh AD, Galea J, Dénes A, Tyrrell PJ, Rothwell NJ. Rapid brain penetration of interleukin-1 receptor antagonist in rat cerebral ischaemia: pharmacokinetics, distribution, protection. Br J Pharmacol 2010; 160: 153-159.

43 Clark SR, McMahon CJ, Gueorguieva I, Rowland M, Scarth S, Georgiou R et al. Interleukin-1 receptor antagonist penetrates human brain at experimentally therapeutic concentrations. J Cereb Blood Flow Metab 2008; 28: 387-394.

44 Shavit Y, Wolf G, Goshen I, Livshits D, Yirmiya R. Interleukin-1 antagonizes morphine analgesia and underlies morphine tolerance. Pain 2005; 115: 50-59.

45 Rolan P, Hutchinson M, Johnson K. Ibudilast: a review of its pharmacology, efficacy and safety in respiratory and neurological disease. Expert Opin Pharmacother 2009; 10: 2897-2904.

46 Cho Y, Crichlow GV, Vermeire JJ, Leng L, Du X, Hodsdon ME et al. Allosteric inhibition of macrophage migration inhibitory factor revealed by ibudilast. Proc Natl Acad Sci USA 2010; 107: 11313-11318
47 Gibson LCD, Hastings SF, McPhee I, Clayton RA, Darroch CE, Mackenzie A et al. The inhibitory profile of lbudilast against the human phosphodiesterase enzyme family. Eur J Pharmacol 2006; 538: 39-42.

48 Johnson K, Matsuda K, Iwaki Y. Ibudilast for the treatment of drug addiction and other neurological conditions. Clin Invest 2014; 4: 269-279.

49 Mizuno T, Kurotani T, Komatsu Y, Kawanokuchi J, Kato H, Mitsuma N et al. Neuroprotective role of phosphodiesterase inhibitor ibudilast on neuronal cell death induced by activated microglia. Neuropharmacology 2004; 46: 404-411.

50 Lilius TO, Rauhala PV, Kambur O, Kalso EA. Modulation of morphine-induced antinociception in acute and chronic opioid treatment by ibudilast. Anesthesiology 2009; 111: 1356-1364.

51 Ledeboer A, Liu T, Shumilla JA, Mahoney JH, Vijay S, Gross Ml et al. The glial modulatory drug AV411 attenuates mechanical allodynia in rat models of neuropathic pain. Neuron Glia Biol 2006; 2: 279-291.

52 Islam AK, Cooper ML, Bodnar RJ. Interactions among aging, gender, and gonadectomy effects upon morphine antinociception in rats. Physiol Behav 1993; 54: 45-53.

53 Liu L, Coller JK, Watkins LR, Somogyi AA, Hutchinson MR. Naloxone-precipitated morphine withdrawal behavior and brain IL-1 $\hat{I}^{2}$ expression: Comparison of different mouse strains. Brain Behav Immun 2011; 25: 1223-1232.

54 Headache Classification Committee of the International Headache Society. New appendix criteria open for a broader concept of chronic migraine. Cephalalgia 2006; 26: 742-746.

(c) (i) $\Theta$ This work is licensed under a Creative Commons AttributionBY NC ND NonCommercial-NoDerivs 4.0 International License. The images or other third party material in this article are included in the article's Creative Commons license, unless indicated otherwise in the credit line; if the material is not included under the Creative Commons license, users will need to obtain permission from the license holder to reproduce the material. To view a copy of this license, visit http:// creativecommons.org/licenses/by-nc-nd/4.0/ 